Palavras chave:

Área degradada

Espécies colonizadoras Sucessão ecológica Serra do Espinhaço

Histórico:

Recebido 22/ I / 20 I I

Aceito 02/10/2014

Keywords:

Degraded area

Colonizing species

Ecological succession

Serra do Espinhaço

Correspondência: cristianyamaral@yahoo.com.br

DOI: I0.1590/0 I04776020152102| 405
Cristiany Silva Amaral', Wander Gladson Amaral', Israel Marinho Pereira', Paula Alves Oliveira', Vinicius de Morais Machado²

\section{COMPARAÇÃO FLORÍSTICO-ESTRUTURAL DOS ESTRATOS ADULTOS E REgENERANTES EM ÁREA MINERADA DE CAMPO RUPESTRE, DIAMANTINA, MG}

RESUMO: Objetivou-se, neste trabalho, comparar a estrutura fitossociológica e verificar a similaridade dos estratos adulto e regenerante em uma antiga lavra de mineração. Para a amostragem da vegetação arbórea, foram alocadas em um transecto de $50 \times 100 \mathrm{~m}, 50$ parcelas de $10 \times 10 \mathrm{~m}$ onde foram amostrados todos os indivíduos vivos com diâmetro a 0,3 $m$ do solo $\geq 3 \mathrm{~cm}\left(D_{30} \geq 3 \mathrm{~cm}\right)$. A regeneração natural foi amostrada em subparcelas de 2 $\times 2 \mathrm{~m} \mathrm{e} 5 \times 5 \mathrm{~m}$, onde foram amostrados plantas com $0, \mathrm{I} \mathrm{m} \leq$ altura $<0,5 \mathrm{~m} \mathrm{e} \mathrm{0,5} \mathrm{m} \leq$ altura $<\mathrm{I}, 50 \mathrm{~m}$, respectivamente, estando as subparcelas localizadas no canto superior direito das 50 parcelas permanentes de $10 \times 10 \mathrm{~m}$, sendo amostrados indivíduos com $D^{2} S_{30} \leq 3 \mathrm{~cm}$ e altura superior a $10 \mathrm{~cm}$. Foram amostrados para as comunidades adultas e regenerantes I 262 indivíduos, distribuídos por 2 I famílias e 45 espécies. O índice de Shannon Weaver $\left(H^{\prime}\right)$, para os estratos adultos e regenerantes foram, respectivamente: 2 , I 8 nats $\cdot$ ind $^{-1}$ e 2,73 nats·ind ${ }^{-1}$. As espécies com o maior índice de regeneração natural foram Lavoisiera montana (14,28\%); Lavoisiera pectinata (14,26\%); Microlicia isophylla (14,13\%); Baccharis elliptica (10,71\%); Baccharis sp I (7,15\%) e Cambessedia menbranaceae (3,63\%). As espécies que se destacaram em relação aos valores de importância no estrato adulto foram Lavoisiera montana (28,59\%); Eremanthus erythropappus (29,98\%); Microlicia isophylla (5,87\%); Pseudobrickellia sp (5,26\%); Baccharis elliptica (5, 18\%) e Palicourea rigida (4,31\%). O índice de similaridade de Jaccard entre o estrato regenerante e adulto foi de $69,56 \%$. $O$ estudo permitiu conhecer as principais diferenças florísticas e estruturais dos estratos estudados contribuindo para o conhecimento das principais espécies colonizadoras desse ambiente.

\section{FLORISTIC-STRUCTURAL COMPARISON OF ADULTS AND REGENERATING STRATA IN A MINED AREA OF CAMPO RUPESTRE, DIAMANTINA, MG}

ABSTRACT: The objective of this work was to compare the phytosociological structure and to verify the similarity of the adult and regenerating stratum in an old mining site. For the arboreal vegetation sampling, 50 plots of $10 \times 10 \mathrm{~m}$ were placed in a transect of $50 \times$ $100 \mathrm{~m}$, where all of the living individuals with $0.3 \mathrm{~m}$ diameter from the soil $\geq 3 \mathrm{~cm}\left(\mathrm{DAS}_{30}\right.$ $\geq 3 \mathrm{~cm}$ ) were sampled. The natural regeneration was sampled in sub plots of $2 \times 2 \mathrm{~m}$ and $5 \times 5 \mathrm{~m}$ for plants with $0.1 \mathrm{~m} \leq$ height $<0.5 \mathrm{~m}$ and $0.5 \leq$ height $<1.5 \mathrm{~m}$, respectively. The sub portions were located in the superior right corner of the 50 permanent 10 x $10 \mathrm{~m}$ plots where individuals with $D^{-S_{30}} \leq 3 \mathrm{~cm}$ and height superior to $10 \mathrm{~cm}$ were sampled. 1262 individuals were sampled for the adult and regenerating communities, spread in 21 families and 45 species. The index of Shannon Weaver ( $\left.H^{\prime}\right)$, for the adult and regenerating stratums was: 2.18 nats.ind ${ }^{-1}$ and 2.73 nats.ind ${ }^{-1}$, respectively. The species with the larger natural regeneration index were Lavoisiera montana (14.28\%); Lavoisiera pectinata (14.26\%); Microlicia isophylla (14.13\%); Baccharis elliptica (I0.7I\%); Baccharis sp I (7.15\%) and Cambessedia menbranaceae (3.63\%). The species that presented the largest values of importance in the adult stratum were Lavoisiera montana (28.59\%); Eremanthus erythropappus (29.98\%); Microlicia isophylla (5.87\%); Pseudobrickellia sp (5.26\%); Baccharis elliptica (5.18\%) and Palicourea rigida (4.31\%). The Jaccard index of similarity between the regenerating and the adult stratums was $69.56 \%$. This study identified the main floristic and structural differences of the studied stratums, contributing to the knowledge of the main species that colonize this environment.

' Universidade Federal dos Vales do Jequitinhonha e Mucuri - Diamantina, Minas Gerais, Brasil

2 Universidade de Brasília - Brasília, Distrito Federal, Brasil 


\section{INTRODUÇÃO}

A Serra do Espinhaço é uma cadeia montanhosa que atravessa de norte a sul os estados da Bahia e de Minas Gerais. As quotas altitudinais variam, aproximadamente, entre 700 e 2000 m acima do nível do mar e seu ambiente montanhoso representa um centro de concentração de biodiversidade e endemismos (HARLEY, 1995). Nesse contexto, destacam-se os campos rupestres que, apesar da alta biodiversidade, importância conservacionista e econômica, muitas de suas espécies estão em vias de extinção, em razão da pequena área que ocupam e da forte ação antrópica que vêm sofrendo (MENEZES; GIULIETTI, 2000).

Dentre os fatores de perturbação, chama a atenção o alto grau de impacto que as intensas atividades de mineração promovem, pois as áreas deixadas com subsolo ou cascalho expostos permanecem destituídas de vegetação, mesmo tendo sido abandonadas por décadas (MENEZES; GIULIETTI, 2000). Assim, o conhecimento do processo de regeneração paralelamente a estudos florísticos e fitossociológicos de longa duração nesses ambientes pode possibilitar 0 entendimento sobre a manutenção da diversidade (FELFILI, 1997), estabilidade e dinâmica de comunidades, além de oferecer subsídios técnicos às ações voltadas para conservação, preservação, manejo e recuperação dessas fitofisionomias.

Como os estudos dos estratos regenerantes e adultos para a vegetação de campo rupestre ainda são incipientes, há a necessidade de se realizar um maior número de estudos florísticos e da estrutura da vegetação colonizadora, tendo em vista que a carência atual de estudos não permite análises comparativas consistentes.

Com isso, neste trabalho, objetivou-se comparar a estrutura fitossociológica e verificar a similaridade dos estratos adulto e regenerante, permitindo, assim, conhecer espécies potenciais para serem usadas em programas de recuperação de áreas degradadas em ambiente de campo-rupestre.

\section{MATERIAL E MÉTODOS}

O estudo foi realizado em uma área de campo rupestre, no Parque Estadual do Biribiri, Diamantina, Minas Gerais, na Serra do Espinhaço Meridional situada nas coordenadas UTM 649039,72E e 7987046,96S. O parque foi criado em setembro de 1988 e encontra-se sobre a Formação Sopa-Brumadinho, uma das formações basais do Supergrupo Espinhaço. A classe de solo predominante é o Neossolo Quartzarênico Órtico típico composto quase que exclusivamente do mineral quartzo que, além de quase insolúvel, promove a formação de solos arenosos e rasos (ALMEIDA-ABREU et al., 2005). A cobertura vegetal típica desse pedoambiente é o cerrado rupestre e o campo rupestre, ecótipos que apresentam elevado déficit hídrico sazonal (INSTITUTO ESTADUAL DE FLORESTAS - IEF, 2004), com predomínio de manchas de floresta estacional semidecidual. O clima é do tipo Cwb, segundo a classificação de Köppen, I,390 $\mathrm{m}$ de altitude, temperatura média anual variando de $18^{\circ}$ a $19^{\circ} \mathrm{C}$, precipitação média anual na faixa de I,250 a I,550 $\mathrm{mm}$ e umidade relativa do ar de $75,6 \%$ (ALMEIDAABREU et al., 2005).

Para o estudo quali-quantitativo da comunidade arbustivo-arbórea selecionou-se uma área de lavra de mineração (garimpo) de diamante desativada minerada, segundo dados do Instituto Estadual de Florestas (IEF) de Diamantina (dados não publicados), no período colonial e não há relatos de atividades antrópicas, posteriormente à mineração.

Para a amostragem da vegetação, foram alocadas, em um transecto de $50 \times 100 \mathrm{~m}, 50$ parcelas de $10 \times 10 \mathrm{~m}\left(100 \mathrm{~m}^{2}\right)$, totalizando uma área amostral de $5,000 \mathrm{~m}^{2}$. A regeneração natural foi amostrada, em subparcelas de $5 \times 5 \mathrm{~m}$ e $2 \times 2 \mathrm{~m}$, localizadas no canto superior direito das 50 parcelas permanentes de 10 x $10 \mathrm{~m}$. Foi usado o método das parcelas de tamanho diferenciado para cada uma das classes de tamanho, adaptação da metodologia adotada por Finol (197I). As classes de tamanho da RN adaptadas e adotadas foram as seguintes: Classe I - plantas com $0,1 \mathrm{~m} \leq$ altura $<$ $0,5 \mathrm{~m}$; Classe II - plantas com 0,5 $\mathrm{m} \leq$ altura $<\mathrm{I}, 50$ I,50 m; Classe III - plantas com altura $\geq 1,50 \mathrm{~m}$. Para a classe I foram adotadas parcelas de $2 \times 2 \mathrm{~m}\left(4 \mathrm{~m}^{2}\right)$ e para as classes II e III parcelas de $5 \times 5 \mathrm{~m}\left(25 \mathrm{~m}^{2}\right)$. O sistema de classificação utilizado para a identificação botânica das plantas foi o APG II (ANGIOSPERM PHYLOGENY GROUP - APG, 2003).

Foram identificadas e mensuradas as alturas das espécies da comunidade arbórea com diâmetros a 30 $\mathrm{cm}$ do solo superior a $3 \mathrm{~cm}$. Os parâmetros utilizados para análise da estrutura da regeneração natural foram densidade, frequência, classe de tamanho absoluta e relativa, e índice de regeneração natural (FINOL, I97I). A composição florística da regeneração foi comparada ao levantamento da comunidade adulta pelo índice de similaridade de Jaccard (Sj).

A estrutura da comunidade arbórea $\left(\mathrm{DAS}_{30} \geq\right.$ $3 \mathrm{~cm}$ ) foi descrita por meio dos cálculos dos parâmetros fitossociológicos: densidade, frequência e dominâncias absoluta e relativa e o índice de valor de importância (IVI), além da diversidade e equabilidade (índices de Shannon- 
Wiener e Pielou) (JACOBI et al., 2008; MESSIAS et al., 2012; MUELLER-DOMBOIS; ELLENBERG, 1974).

\section{RESULTADOS E DISCUSSÃO}

Foram inventariados na área minerada para as comunidades regenerantes e adultas um total de
21 famílias e 45 espécies representados por 1262 indivíduos. Dos indivíduos amostrados, alguns não foram identificados em nível de espécies por falta de material botânico adequado, totalizando 50 indivíduos representando $3,96 \%$ do total amostrado (Tabela I).

Para a regeneração natural, encontraram-se 1066 indivíduos distribuídos em I 9 famílias e 39 espécies.

TABELA 1 Relação das famílias e espécies identificadas, em diferentes ambientes, em uma área de campo rupestre no Parque Estadual do Biribiri, Diamantina, MG. Em que: $A D$ = estrato adulto; $R N=$ estrato de regeneração natural; $T$ = número total de indivíduos da i-ésima espécie.

TABLE 1 List of families and species identified in different environments of a campo rupestre area in Biribiri State Park. Where: AD $=$ adult strata; $\mathrm{RN}=$ natural regeneration strata, $\mathrm{T}=$ total number of individuals of the $\mathrm{i}$-th species.

\begin{tabular}{|c|c|c|c|c|c|c|c|}
\hline \multirow{2}{*}{ Família / espécie } & \multicolumn{2}{|c|}{ Estrato (NI) } & \multirow{2}{*}{$\mathrm{T}$} & \multirow{2}{*}{ Família / espécie } & \multicolumn{2}{|c|}{ Estrato $(\mathrm{NI})$} & \multirow{2}{*}{$\mathrm{T}$} \\
\hline & $A D$ & RN & & & $A D$ & RN & \\
\hline Aquifoliaceae & & & & Malpighiaceae & & & \\
\hline Ilex affinis Gardn. & 1 & -- & 1 & Heteropteris sp. & -- & 1 & 1 \\
\hline Araliaceae & & & & Melastomataceae & & & \\
\hline Schefflera angustissima (March.) & -- & 3 & 3 & Bellucia sp. & -- & 21 & 21 \\
\hline Asteraceae & & & & Cambessedia membranaceae DC. & 1 & 35 & 36 \\
\hline Baccharis dentata (Vell.) G.M.Barroso. & -- & 13 & 13 & Lavoisiera Montana Cogn. & 66 & 159 & 225 \\
\hline Baccharis elliptica Gardner. & 10 & 121 & 131 & Lavoisiera pectinata Cogn. & -- & 168 & 168 \\
\hline Baccharis sp 1 & 7 & 74 & 81 & Marcetia sp. & -- & 34 & 34 \\
\hline Baccharis tridentata Vahl. & -- & 3 & 3 & Miconia pepericarpa DC. & -- & 28 & 28 \\
\hline Eremanthus erythropappus (DC.) & 49 & 18 & 67 & Miconia sp. & -- & 5 & 5 \\
\hline Eremanthus incanus (Less.) Less. & -- & 1 & 1 & Microlicia isophyla. & 12 & 137 & 149 \\
\hline Lychnophora ericoides Less. & -- & 15 & 15 & Tibouchina candolleana (Mart) DC & 6 & 7 & 13 \\
\hline Pseudobrickellia sp. & 9 & 5 & 14 & Tibouchina granulosa Cogn. & 2 & -- & 2 \\
\hline Trembleya parviflora (D.Don) Cogn. & 5 & 2 & 7 & Tibouchina sellowiana (Cham.) Cogn & -- & 3 & 3 \\
\hline Boraginaceae & & & & Myrcinaceae & & & \\
\hline Cordia rufescens A.DC. & 1 & 1 & 2 & Myrsine guianensis (Aubl.) Kuntze. & 3 & 6 & 9 \\
\hline Clusiaceae & & & & Myrtaceae & & & \\
\hline Clusia criuva Cambess. & -- & 2 & 2 & Myrcia guianensis (Aubl.) DC. & -- & 1 & 1 \\
\hline Kielmeyera lathrophyton Saddi. & 1 & & 1 & Siphoneugena densiflora O.Berg. & -- & 8 & 8 \\
\hline Ericaceae & & & & Pentaphylacaceae & & & \\
\hline Agorista sp. & -- & 2 & 2 & Ternstroemia brasiliensis Cambess. & 1 & -- & 1 \\
\hline Erythroxylaceae & & & & Phyllanthaceae & & & \\
\hline Erythroxylum buxus Peyr. & 1 & -- & 1 & Richeria gramois Vahl. & 1 & -- & 1 \\
\hline Erythroxylum cuneifolium. Schult & -- & 11 & 11 & Polypodiaceae & & & \\
\hline Erythroxylum suberosum A.St.-Hil. & 3 & 3 & 6 & Pteridium aquilinum. L. Kuhn. & -- & 21 & 21 \\
\hline Fabaceae & & & & Rubiaceae & & & \\
\hline Chamaecrista $s p$. & -- & 48 & 48 & Palicourea rigida Kunth. & 9 & 10 & 19 \\
\hline Lamiaceae & & & & Sapindaceae & & & \\
\hline Hyptis sp. & -- & 3 & 3 & Cupania vernalis Cambess. & 1 & 6 & 7 \\
\hline Lauraceae & & & & Solanaceae & & & \\
\hline Ocotea pomaderroides (Meissn.) Mez. & -- & 5 & 5 & Aureliana sp. & 3 & 1 & 4 \\
\hline Malpighiaceae & & & & Verbenaceae & & & \\
\hline Byrsonima crassa Nied. & -- & 6 & 6 & Lippia sp. & -- & 14 & 14 \\
\hline Byrsonima intermedia A.Juss. & 3 & 16 & 19 & Sem identificação & 1 & 49 & 50 \\
\hline Total parcial & 90 & 352 & 442 & Total parcial & 106 & 714 & 820 \\
\hline
\end{tabular}


Esses valores foram inferiores aos encontrados por Pinto et al. (2009), estudando a composição florística de um cerrado rupestre em Cocalzinho de Goiás, Goiás, com 36 famílias e 65 espécies. No estrato adulto, foram registrados 196 indivíduos pertencentes a 14 famílias e 23 espécies. Em outras áreas de mesma fisionomia foram encontrados números de famílias e espécies superiores aos encontrados neste trabalho, respectivamente: 30 e 56 no Parque Estadual dos Pirineus (MOURA et al., 2007); 16 e 28 em afloramento rochoso granítico de altitude no Parque Estadual da Serra do Brigadeiro, Minas Gerais, Brasil (CAIAFA; SILVA, 2007); 23 e 54 no Parque Estadual da Serra do Brigadeiro (CAIAFA; SILVA, 2005).

Como era de se esperar, a população de regenerantes é muito superior à adulta (Tabela I). Esse resultado é um indicador da capacidade de autorregeneração dessa comunidade e a estrutura da mesma dependerá das condições favoráveis ao desenvolvimento dos indivíduos presentes na regeneração.

Essa situação parece indicar também que, tanto herbívoros quanto patógenos desempenham um papel importante sobre os regenerantes, sem que, contudo, possam ser considerados fatores limitantes para a população de plântulas e indivíduos jovens (CONNELL, 197I; JANZEN, 1970). Esse processo depende, inicialmente, da dispersão de sementes, que devem apresentar viabilidade, escapar de predadores e encontrar condições adequadas à germinação (GOMÉZPOMPA et al., |991). Posteriormente, a sobrevivência e desenvolvimento dos indivíduos da regeneração dependem de fatores fenológicos, genéticos e ambientais (FELFILI, 1997) e de distúrbios ocasionais, como fogo (HOFFMANN, 1998; SOARES et al., 2006). Todos esses fatores produzem dinâmicas nas condições de crescimento e interferem na estrutura da comunidade, favorecendo diferentes espécies ao longo do tempo (MEDEIROS et al., 2007).

A composição florística da área, para o estrato adulto e regenerante, compreendeu 22 famílias e 45 espécies. Dessas espécies, 16 ocorreram nos dois estratos, 23 ocorreram apenas na regeneração e seis foram exclusivas do estrato adulto (Tabela I). Em relação às famílias, das 14 espécies presentes na regeneração, oito não estão representadas no estrato adulto, sendo elas Araliaceae, Ericaceae, Fabaceae, Lamiaceae, Lauraceae, Myrtaceae, Polypodiaceae e Verbenaceae.

As famílias que mais contribuíram com a riqueza florística nos estratos de regeneração e adulto, foram:
Melastomataceae (I I), Asteraceae (nove), Malpighiaceae (três) e Erythroxylaceae (três).

Em Asteraceae as espécies Baccharis dentata, Baccharis tridentata, Eremanthus incanus e Lychnophora ericoides são exclusivas da regeneração, enquanto que nenhuma espécie foi exclusiva do estrato adulto.

Para Melastomataceae, a espécie Tibouchina candoleana é exclusiva do estrato arbóreo-arbustivo, enquanto Bellucia sp, Lavoisiera pectinata, Marcetia sp, Miconia pepericarpa, Miconia sp e Tibouchina sellowiana são exclusivas da regeneração. Resultados semelhantes são relatados em outras áreas de mesma fitofisionomia (RAPINI et al., 2008).

Em uma área de cerrado rupestre em Brasília, DF, amostrada por Amaral et al. (2006), as famílias Fabaceae (nove), Melastomataceae e Malpighiaceae (quatro cada) foram as mais ricas em espécies.Dentre as 45 espécies registradas neste estudo, Lavoisiera montana (225) foi a que apresentou maior número de indivíduos amostrados, seguida de lavoisiera pectinata (168), Microlicia isophyla (149), Baccharis elliptica (I3I), Baccharis sp I (8I), Eremanthus erythropappus (67), Chamaecrista sp (48) e Cambessedia membranaceae (36) representando, no total, $65,10 \%$ dos indivíduos em regeneração.

As espécies Erythroxylum buxus, Ilex affinis, Kielmeyera lanthrophyton, Richeria gramois, Tibouchina candoleana e Kielmeyera lanthrophyton são exclusivas do estrato adulto. Isso sugere que elas podem ter aparecido, em razão do recrutamento de indivíduos que estavam presentes na regeneração dos anos anteriores (FELFILI et al., 2007). Segundo Hoffmann (1998), a ausência de famílias e espécies no estrato de regeneração, presentes no estrato adulto, pode ser atribuída a fatores biológicos das populações.

As espécies das famílias Melastomataceae e Asteraceae foram as mais representativas na área de estudo, o que pode ser explicado pela particularidade de seus frutos serem secos e leves, facilmente dispersos pelos ventos (MIRANDA, 2005). A eficiência da dispersão anemocórica das espécies da família Asteraceae também tem sido enfatizada para a disseminação de propágulos, em áreas antropizadas como clareiras, bordas de matas, pastagens e áreas degradadas, o que evidencia a importância do uso de espécies dessa família nos projetos de recuperação de áreas degradadas (HEIDEN et al., 2007).

As espécies Baccharis elliptica; Baccharis sp I; Eremanthus erythropappus; Pseudobrickellia sp; Byrsonima intermedia; Cambessedia membranaceae; Lavoisiera 
montana; Microlicia isophylla; Cordia rufescens; Trembleya parviflora, Palicourea rigida; Cupania vernalis; Aureliana sp; Erythroxylum suberosum; foram registradas em ambos os estratos. Os gêneros Eremanthus e Baccharis detêm espécies com grande eficiência na colonização de ambientes antropizados. Araújo et al. (2006) também destaca a importância do uso da candeia (Eremanthus eritropappus) na recuperação de áreas degradadas.

As espécies Eremanthus incanus e Miconia sp ocorreram apenas na regeneração natural. Em estudos realizados por Nappo et al. (2000), em uma área minerada no município de Poços de Caldas, as espécies do gênero Miconia foram as que apresentaram melhor desempenho na colonização e estruturação da regeneração natural, sendo indicadas também como espécies potenciais para uso nos programas de recuperação de áreas mineradas em condições semelhantes sobre a estratégia sucessional.

O índice de Shannon Weaver para a regeneração natural $\left(H^{\prime}=2,73\right)$ foi superior ao encontrado para a vegetação adulta $\left(H^{\prime}=2,18\right)$. Segundo Barreira et al. (2007) valores baixos de H' podem ser explicados pelo fato do componente arbustivo-arbóreo do cerrado ser heliófito e, à medida que existe um maior sombreamento dos estratos inferiores, a diversidade diminui de forma sensível para esse componente. No entanto, esse comportamento não foi evidenciado no presente estudo, uma vez que o fator luz nas áreas mineradas não é considerado limitante.

Os valores do índice de equabilidade de Pielou (J') foram: 0,69 e 0,7I para os estratos adultos e em processo de regeneração natural, respectivamente. Assim, podemos inferir que existe um equilíbrio entre a concentração de espécies dominantes e a concentração de espécies não-dominantes (PIELOU, 1966).

Com relação ao índice de Jaccard, encontrouse alta similaridade florística entre $o$ estrato de regeneração natural e adulto, com valores em torno de $69,56 \%$ (16 espécies em comum), os quais são considerados altos quando superiores a 0,5 (FELFILI; RESENDE, 2003). Espera-se que uma comunidade em equilíbrio mantenha sua composição florística e estrutural ao longo do tempo, de modo que a similaridade florística da regeneração natural com o estrato lenhoso pode ser um indicador de estabilidade da comunidade (MEDEIROS et al., 2007).

Para a regeneração natural foram amostrados 1066 indivíduos, em 0,125 ha. Em relação ao estrato adulto foram identificados 196 indivíduos em 0,5 ha. A densidade absoluta para a regeneração natural foi estimada em 8528 ind.ha-1, e para o estrato adulto em 392 ind $\cdot$ ha $^{-1}$. Esse resultado é um indicador da capacidade de autorregeneração dessa comunidade e a estrutura da mesma dependerá das condições favoráveis ao desenvolvimento dos indivíduos presentes na regeneração (MEDEIROS et al., 2007).

A espécie Lavoisiera pectinata foi a que apresentou a maior densidade absoluta ( 344 indivíduos. ha $^{-1}$ ), o que corresponde a $15,76 \%$ da densidade total. A maior concentração de indivíduos nessa área (87,5\%) foi registrada na classe de tamanho 2 , seguida da classe de tamanho I (8,3\%), e o restante $(4,2 \%)$ estava distribuído na classe de tamanho 3 (Figura I).

A maior concentração de indivíduos de pequeno porte nas áreas avaliadas é condizente, uma vez que esta é a distribuição da altura esperada para

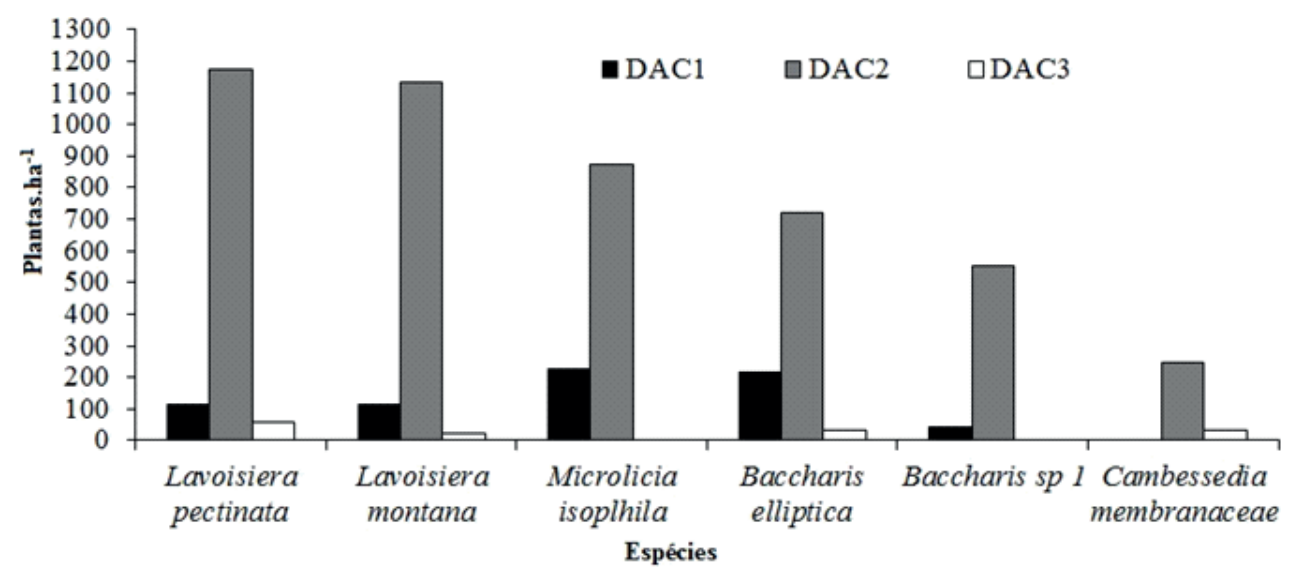

FIGURA 1 Número de planta por hectare por classe de tamanho da regeneração natural em uma área minerada em ambiente de campo rupestre no Parque Estadual do Biribiri, Diamantina, MG.

FIGURE 1 Number of plants per hectare by size class of natural regeneration in a mined area in the campo rupestre in Biribiri State Park, Diamantina, MG. 
uma área coberta por vegetação de diferentes espécies, de idades desconhecidas, e em estágio de regeneração. Entretanto, sugere-se que para a regeneração uma redução no número de indivíduos e espécies nas maiores classes de altura é esperada, em razão da capacidade de suporte do ambiente, pois a maior parte dos indivíduos da regeneração não irá se estabelecer, em decorrência da competição (MEDEIROS et al., 2007).

$\mathrm{Na}$ área minerada, as espécies com o maior índice de regeneração natural foram Lavoisiera montana (I4,28\%); Lavoisiera pectinata (14,26\%); Microlicia isophylla (14,13\%); Baccharis elliptica (10,71\%); Baccharis sp I (7,15\%); Cambessedia menbranaceae $(3,63 \%)$ (Figura 2). Pode-se inferir que a natureza propiciou grande facilidade para seu estabelecimento na área, o que torna essas espécies interessantes para serem utilizadas em programas de recuperação de áreas degradadas.

As espécies que se destacaram em relação aos valores de importância no estrato adulto foram Lavoisiera montana (29,98\%); Eremanthus erythropappus (28,59\%); Microlicia isophylla (5,87\%); Pseudobrickellia sp (5,26\%); Baccharis elliptica $(5,18 \%)$ e Palicourea rigida (4,31\%) (Figura 3), podendo ser consideradas as espécies que obtiveram maior sucesso na exploração dos recursos daquele ambiente.

Verifica-se que Lavoisiera montana e Eremanthus erythropappus apresentam os maiores valores de importância. Isso indica que essas espécies possuem populações autorregenerativas e adaptadas à área em estudo, podendo ser utilizadas nos programas de recuperação em ecossistemas similares.

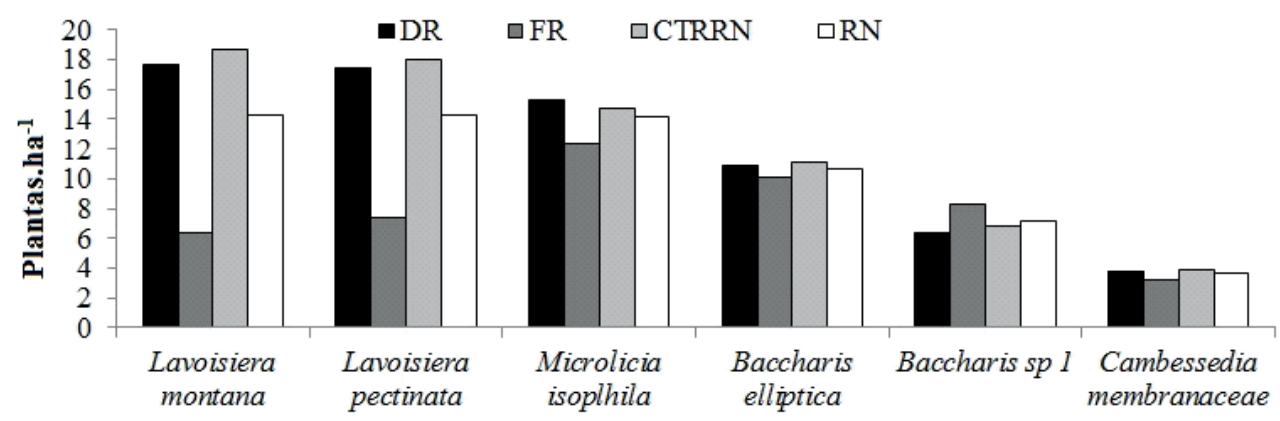

Espécies

FIGURA 2 Parâmetros da regeneração natural para as seis espécies do estrato regenerante em uma área minerada de campo rupestre, situada no Parque Estadual do Biribiri, Diamantina, MG. Onde: densidade relativa (DR), frequência relativa $(F R)$, classe de tamanho relativa da regeneração natural (CTRRN) e índice de regeneração (RN).

FIGURE 2 Regeneration index of the six species of the natural regeneration strata in a mined area in the campo rupestre in Biribiri State Park, Diamantina, MG. Where: relative density (DR), relative frequency (FR), relative class size of the natural regeneration (CTRRN) and regeneration index $(R N)$.

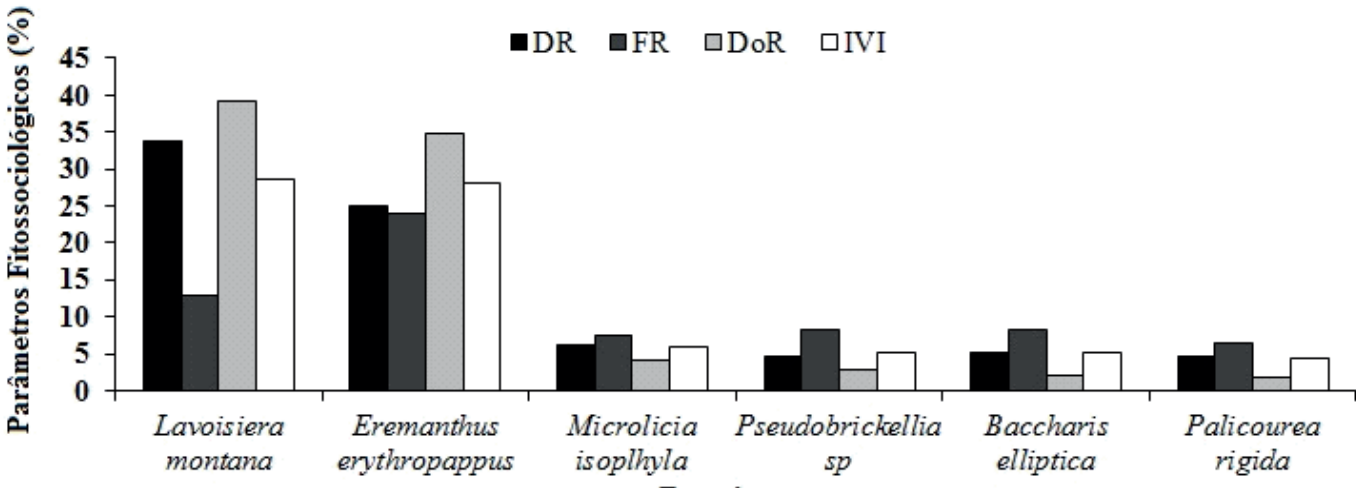

Espécies

FIGURA 3 Valores dos parâmetros fitossociológicos relativos para o estrato arbóreo em ambiente de campo rupestre no Parque Estadual do Biribiri, Diamantina, MG, em que: DR = densidade relativa; FR = frequência relativa; DoR = dominância relativa e IVI = índice de valor de importância (IVI).

FIGURE 3 Relative values of the phytosociological parameters for the arboreal stratum in a campo rupestre in Biribiri State Park, Diamantina, MG, where: relative density = DR; relative frequency = FR; relative dominance $=$ DoR and importance value index (IVI). 


\section{CONCLUSÕES}

Com base nos resultados obtidos pode-se concluir que: há uma elevada similaridade entre os estratos adulto e regenerante na área em estudo; as espécies Lavoisiera montana; Eremanthus erythropappus; Microlicia isophylla; Pseudobrickellia sp.; Baccharis elliptica, Tibouchina candolleana e Palicourea rigida se destacaram, tanto na regeneração natural quanto no estrato adulto, podendo ser consideradas potenciais para uso na recuperação de ecossistemas congêneres; e as espécies Pseudobrickellia sp., e Eremanthus erythropappus apresentaram maior população no estrato das adultas e um decréscimo na regeneração natural, enquanto que, Lavoisiera Montana, Microlicia isophyla e Baccharis elliptica apresentaram comportamento inverso, com elevada população na regeneração natural e elevada queda no estrato adulto.

\section{REFERÊNCIAS}

ALMEIDA-ABREU, P. A.; FRAGA, L. M. S.; NEVES, S. C. Geologia. In: SILVA; A. C.; PEDREIRA, L. C. V. S. F; ALMEIDA-ABREU, P. A. (Org.). Serra do Espinhaço Meridional: paisagens e ambientes. Diamantina: UFVJM, 2005. v. I, p. 17-44.

AMARAL, A. G.; PEREIRA, F. F. O.; MUNHOZ, C. B. R. Fitossociologia de uma área de cerrado rupestre na Fazenda Sucupira, Brasília-DF. Cerne, Lavras, v. 12, n. I, p. 350-359, 2006.

ANGIOSPERM PHYLOGENY GROUP. An update of the angiosperm phylogeny group classification for the orders and families of flowering plants: APG II. Botanical Journal of the Linnean Society, London, v. I4 I, p. 399-436, 2003.

ARAÙJO, F. S.; MARTINS, S. V.; LANI, J. L.; PIRES, I. E. Estrutura da vegetação arbustivo-arbórea colonizadora de uma área degradada por mineração de caulim, Brás Pires, MG. Revista Árvore, Viçosa, v. 30, n. I, p. 107-I I6, jan./fev. 2006.

BARREIRA, S.; SCOLFORO, J. R. S.; BOTELHO, S. A.; MELLO, M. J. de. Estudo da estrutura da regeneração natural e da vegetação adulta de um cerrado senso stricto para fins de manejo florestal. Scientia Forestalis, Santa Maria, n. 6I, p. 64-78, jun. 2007.

CAIAFA, A. N.; SILVA, A. F. Composição florística e espectro biológico de um Campo de Altitude no Parque Estadual da Serra do Brigadeiro, Minas Gerais, Brasil. Rodriguésia, Rio de Janeiro, v. 56, n. 87, p. I63-173, 2005.

CAIAFA, A. N.; SILVA, A. F. Strutural analysis of the vegetation on a highland granitic rock outcrop in Southeast Brazil. Revista Brasileira de Botânica, São Paulo, v. 30, n. 4, p. 657-664, out. 2007.
CONNELL, J. H. On the role of natural enemies in preventing competitive exc l usion in some marine animals and in rain forest trees. In: BOEN, P.J. den; GRADWELL, G. R. (Ed.). Dynamics of populations. Wageningen: Centre of Agricultural Publishing and Documentations, 197I. p. 298-3/2.

FELFILI, J. M. Dynamics of the natural regeneration in the Gama gallery forest in central Brazil. Forest Ecology and Management, Amsterdam, v. 9I, n. 2/3, p. 235-245, 1997.

FELFILI, J. M.; REZENDE, A. V.; SILVA JÚNIOR, M. C.; SILVA, P. E. N.; WALTER, B. M. T.; ENCINAS, J. I.; SILVA, M. A. Fitossociologia da vegetação arbórea. In: FELFILI, J. M.; REZENDE, A. V.; SILVA JÚNIOR, M. C. (Org.). Biogeografia do bioma Cerrado: vegetação e solos da Chapada dos Veadeiros. Brasília: UnB, 2007. p. 45-96.

FELFILI, J. M.; RESENDE, R. P. Conceitos e métodos em fitossociologia. Brasília: Universidade Federal de Brasília, 2003. (Comunicações-Técnicas Florestais, 5).

FINOL, H. Nuevos parâmetros a considerar-se en el análises estructural de las selvas virgenes tropicales. Revista Forestal Venezoelana, Merida, v. I4, n. 2 I, p. 24-42, 197 I.

GOMÉZ-POMPA, A.; WHITMORE, T. C.; HADLEY, M. Rain forest regeneration and management: man and the biosphere series. Paris: UNESCO; Parthenon Group, I99I.

HARLEY, R. M. Flora of the Pico das Almas, Chapada Diamantina-Bahia, Brasil. In: STANNARD, B. L. E. (Ed.). The trustees of the Royal Botanic Gardens. Kew: Royal Botanic Gardens, 1995. p. 853-86I.

HEIDEN, G.; IGANCI, J. R. V.; BOBROWSKI, V. L.; MACIAS, L. Biogeografia de Baccharis sect. caulopterae (asteraceae) no Rio Grande do Sul, Brasil. Rodriguésia, Rio de Janeiro, v. 58, n. 4, p. 787-796, 2007.

HOFFMANN, W. A. Post-burn reproduction of woody plants in a neotropical savanna: the relative importance of sexual and vegetative reproduction. Journal of Applied Ecology, Oxford, v. 35, n. 7, p. 422-433, 1998.

INSTITUTO ESTADUAL DE FLORESTAS. Plano de manejo parque estadual do Biribiri. Curitiba, 2004.

JACOBI, C. M.; CARMO, F. F.; VINCENT, R. C. Estudo fitossociológico de uma comunidade vegetal sobre canga como subsídio para a reabilitação de áreas mineradas no quadrilátero ferrífero, MG. Revista Árvore, Viçosa, MG, v. 32, p. 345-353, 2008.

JANZEN, D. H. Herbivores and the number of tree species in tropical forests. American Naturalist, Chicago, v. 104, p. 50I-528, 1970.

MEDEIROS, M. M.; FELFILI, J. M.; LÍBANO, A. M. Comparação florístico-estrutural dos estratos de regeneração e adulto em cerrado sensu stricto no Brasil central. Cerne, Lavras, v. I3, n. 3, p. 29I-298, jul./set. 2007. 
MENEZES, N. L.; GIULIETTI, A. M. Campos rupestres. In: MENDONÇA, M. P.; LINS, L. V. (Ed.). Lista vermelha das espécies ameaçadas de extinção da flora de Minas Gerais. Belo Horizonte: Fundação Biodiversitas; Fundação Zoobotânica de Belo Horizonte, 2000. p. 65-73.

MESSIAS, M. C. T. B.; LEITE, M. G. P.; MEIRA-NETO, J. A.; ALVES, K.; RODRIGUES, A. Fitossociologia de campos rupestres quartzíticos e ferruginosos no Quadrilátero Ferrífero, Minas Gerais. Acta Botanica Brasílica, Porto Alegre, v. 26, p. 230-242, 2012.

MIRANDA, C. S. Tipologia de frutos e síndromes de dispersão de uma comunidade de campo rupestre no Parque Estadual da Serra dos Pireneus, Goiás. Goiânia: UEG, 2005.

MOURA, I. O.; KLEIN, V. L.; FELFILI, J. M.; FERREIRA, H. D. Fitossociologia da comunidade lenhosa de uma área de cerrado rupestre no Parque Estadual dos Pireneus, Pirenópolis, Goiás. Revista de Biologia Neotropical, Goiânia, v. 4, n. 2, p. 83-100, 2007.

MUELLER-DOMBOIS, D.; ELLENBERG, H. Aims and methods of vegetation ecology. New York: J. Wiley, 1974. $547 \mathrm{p}$.
NAPPO, M. E.; FONTES, M. A. L.; OLIVEIRA-FILHO, A. T. Regeneração natural em sub-bosque de povoamentos homogêneos de Mimosa scabrella Bentham, implantados em áreas mineradas, em Poços de Caldas, Minas Gerais. Revista Árvore, Viçosa, v. 24, n. 3, p. 297-307, maio/jun. 2000.

PIELOU, E. C. Species diversity and pattern diversity in the study of ecological succession. Journal Theory Biology, Cambridge, v. 10, p. 370-383, 1966.

PINTO, J. R.; LENZA, E.; PINTO, A. S. de. Composição floristica e estrutura da vegetação arbustivo-arbórea em um cerrado rupestre, Cocalzinho de Goiás, Goiás. Revista Brasileira de Botânica, São Paulo, v. 32, n. I, p. I-10, 2009.

RAPINI, A.; RIBEIRO, P. L.; LAMBERT, S.; PIRANI, J. S. A flora dos campos rupestres da cadeia do espinhaço. Megadiversidade, Belo Horizonte, v. 4, n. I/2, p. 16-24, dez. 2008.

SOARES, J. J.; SOUZA, M. H. A. O.; LIMA, M. I. S. Twenty years of post-fire plant succession in a cerrado, São Carlos, SP, Brazil. Brazilian Journal Biology, São Paulo, v. 66, n. 2B, p. 587-602, 2006. 